\title{
Design and calculation of sine wave permanent magnet brushless motor for electric vehicle
}

\author{
Wu Jiahua $^{1 *}$, Sun Fengchang ${ }^{1}$, Deng Xiaochuan ${ }^{1}$, Zhang Zhichao ${ }^{1}$, Yu Dongyang ${ }^{1}$, Wang Zheng ${ }^{1}$, Li Zishuo ${ }^{1}$ \\ ${ }^{1}$ Beijing Branch-State Grid Information And telecommunication Group, Beijing, China
}

\begin{abstract}
With the rapid development of the global automobile industry, energy and environmental problems have become increasingly prominent. In order to realize the sustainable development of new energy, automobile industry has become the direction of new energy. Due to the relatively mature energy storage technology, high efficiency of driving motor and mature control mode, electric vehicles have become the main development direction of new energy vehicles. The main work of this paper is to study the characteristics of various driving motors, design a motor which can meet the performance index of pure electric vehicle, and select an appropriate control scheme.
\end{abstract}

\section{Introduction}

At present, with the rapid development of automobile industry, energy and environmental pollution problems are becoming increasingly prominent, and the requirements of automobile emission standards are also increasing. Therefore, all countries have put forward the corresponding new energy vehicle plan to deal with these problems.

China began to develop electric vehicles as early as the 1980s. During the "Tenth Five Year Plan" and "Eleventh Five Year Plan", China's automobile industry developed rapidly and became the world's largest automobile producer and consumer.

With the support of policy, the domestic self-owned brand electric vehicle industry has also developed vigorously, and has launched its own electric or hybrid vehicles. The most representative of these is the Qin hybrid vehicle launched by BYD Company. The hybrid vehicle adopts the permanent magnet synchronous motor drive mode, its acceleration of $0-100 \mathrm{~km} / \mathrm{h}$ only takes $5.1 \mathrm{~s}$. The comprehensive fuel consumption per $100 \mathrm{~km}$ is less than $3 \mathrm{~L}$.

After years of development, China's electric vehicle technology has made great progress, but compared with foreign developed countries, we still have a big gap in motor structure innovation, materials, control strategy, power electronic technology and so on. In order to catch up with and surpass the core technology of automobile, we need to make efforts and innovation in many aspects.

The main work of this project is to design a sine wave permanent magnet brushless motor and carry out simulation analysis. Electric vehicle requires its drive motor to have the advantages of high efficiency, high power density, wide speed regulation and high overload capacity. According to the main performance requirements of an electric vehicle drive motor in engineering practice, a built-in sine wave permanent magnet brushless motor is designed. The parameters are optimized by the software Ansoft Maxwell, and then the finite element analysis is used for simulation verification.

\section{Electromagnetic design of sine wave permanent magnet brushless motor}

\subsection{Stator design}

The design of the stator mainly includes the selection of the main size, the selection of the number of stator slots, the selection and size of stator slots, the design of winding and so on. This is the key part of the magnetic circuit design of sine wave permanent magnet brushless motor.

The main dimensions of sine wave permanent magnet brushless motor are related to a series of parameters such as torque, power, speed and electromagnetic load grade. In addition, the approximate size of the motor can be determined by selecting the electromagnetic load according to the working environment of the motor.

The main dimension relation of motor is:

$$
D_{a}^{2} l_{\delta}=\frac{6.1 p^{t} \times 10^{8}}{a_{i} k_{\Phi} k_{w} A B_{\delta} n_{H}}
$$

Where: ai is the calculated polar arc coefficient, generally $0.6 \sim 0.8 ; \mathrm{K} \Phi$ is the waveform coefficient of the air gap magnetic field; $\mathrm{KW}$ is the winding coefficient; $\mathrm{A}$ is the line load, which is directly related to the loss of the motor. Generally, the value is $60 \sim 350(\mathrm{~A} / \mathrm{cm})$; $\mathrm{B} \delta$ is the magnetic load, generally $5000 \sim 9000$ (GS); $\mathrm{nH}$ is the rated speed of the motor; $\mathrm{P}$ is the calculated power of the motor.

According to the analysis of stator winding selection in the second chapter, we finally choose the fractional slot structure, and then we need to consider the symmetry

*Corresponding author: 472660752@qq.com 
condition of fractional slot winding. In order to obtain symmetrical electromotive force and magnetomotive force, symmetrical armature winding is required. For three-phase motor, the electromotive force of three-phase winding must be equal, and the phase difference is $120^{\circ}$ electrical angle.

In order to obtain symmetrical armature winding, the number of slots $Z$, the number of poles $P$ and the number of phases m must meet the following requirements: (1) $Z$ / $\mathrm{M}=$ integer, (2) $\mathrm{Z} 0 / \mathrm{M}=$ integer, where $\mathrm{z}=\mathrm{z} 0 \mathrm{t}$ and $\mathrm{P}=$ p0t. $T$ is an integer. This relation is called symmetry condition of fractional slot winding.

On the premise of meeting the above conditions, the value of $t$ should be reduced as far as possible, so as to reduce the number of unit motors and the electromagnetic torque ripple of motors.

Considering the above analysis, we choose the structure of 8-pole and 18 slot.

Because sine wave permanent magnet brushless motor is excited by permanent magnet, the air gap magnetic field contains quite a lot of harmonic components. In order to suppress the third harmonic component, the star connection method is adopted for the stator three-phase winding. The rated power of the motor reaches $16 \mathrm{kw}$, and the motor structure of multi pole and few slots is adopted, so the rotor winding adopts double-layer winding, as shown in Fig. 1.
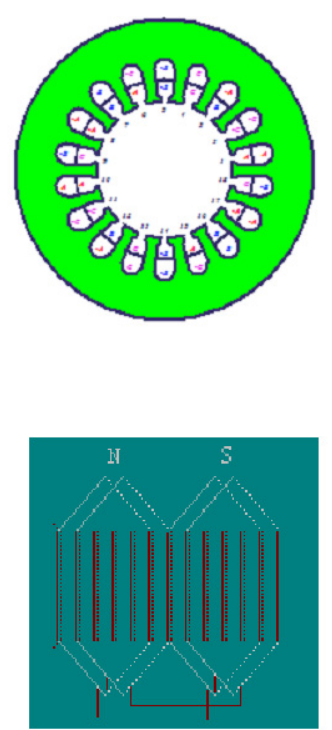

Fig. 1 Stator lamination and stator armature winding

\subsection{Rotor magnetic circuit design}

In the selection of motor rotor structure, the special requirements of electric vehicle for driving motor should be considered. The driving motor of electric vehicle is required to achieve high speed, wide speed range and high power density. As can be seen from the analysis in the second chapter, compared with the surface rotor structure, the built-in rotor has better mechanical strength and is more suitable for high-speed operation, and the anti demagnetization ability of its permanent magnet is also better than that of the surface structure. Therefore, the built-in rotor magnetic circuit structure is selected.
The selection of the number of rotor poles should consider the actual working environment. Generally, with other conditions unchanged, more pole pairs can reduce the size of the motor, save materials, reduce the production cost of the motor, and improve the power density ratio of the motor. But when the number of poles is large, the efficiency of the motor will decline. As an electrically excited synchronous motor, sine wave permanent magnet brushless motor has inherent advantages in efficiency compared with asynchronous motor, so the 8-pole rotor design is selected in this paper.

For the built-in rotor magnetic circuit structure, the main dimension parameters of permanent magnet are magnetization direction length $\mathrm{hm}$, width $\mathrm{bm}$, and axial length $\mathrm{lm}$. In general, the axial length of the permanent magnet is equal to the length of the motor core, so in the actual design, only two other parameters should be considered. For the magnetization direction length hm, we need to choose the appropriate hm value to make the permanent magnet at the best working point. The magnetization direction width bm determines the flux area provided by the permanent magnet, so the performance of the motor is usually adjusted by adjusting bm.

In fact, the permanent magnet design of the motor with built-in radial magnetic circuit structure is very complex, so we need to constantly calculate to get the best working size. The final Rotor magnetic circuit design is shown as Fig. 2.

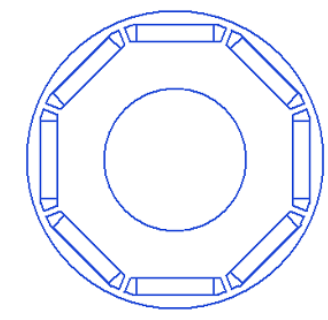

Fig. 2 Rotor structure

\section{Electromagnetic field simulation of sine wave permanent magnet brushless motor}

\subsection{Finite element analysis method}

In the finite element analysis, the complex problem is replaced by the simple problem and then solved. It regards the solution domain as composed of many small interconnected subdomains called finite element, assumes a suitable approximate solution for each element, and deduces the total satisfaction conditions for solving this domain, so as to obtain the solution of the problem. This solution is not an exact solution, but an approximate solution.

In short, the finite element analysis can be divided into three stages: pre-processing, calculation and postprocessing. The pre-processing is to establish the finite element model and complete the mesh generation; the post-processing is to collect the analysis results, so that users can easily extract information and understand the 
calculation results.

\subsection{Simulation modelling of sine wave permanent magnet brushless motor}

After using Ansoft rmxpt software package for preliminary electromagnetic design of the motor, Maxwell $2 \mathrm{D}$ model can be directly output, the model diagram is as follow (Fig. 3).

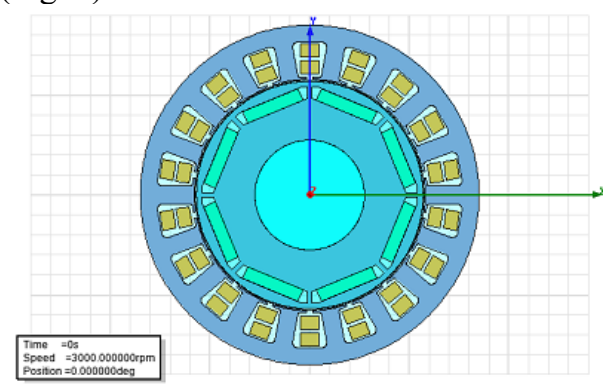

Fig. 3 Maxwell 2D model of motor

The network generation of Maxwell 2D model of motor is the most important step of finite element discretization, and the quality of network generation directly affects the accuracy of finite element analysis results. The software has its own subdividing solver. In most cases, a better subdividing network can be obtained without manual subdividing. The closer the triangulation is to the regular triangle, the better the effect of triangulation is. Finer subdivision leads to better effect of calculation, but longer calculation time. The subdivision diagram is as follow (Fig. 4).

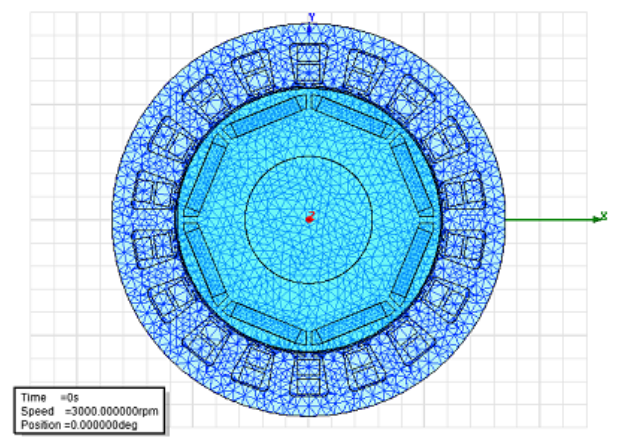

Fig. 4 Subdivision of the motor

\subsection{Analysis of motor magnetic field based on FEM}

When analysing the no-load magnetic field of the motor, the stator armature winding current is zero and only the permanent magnet acts. Through the finite element analysis, the no-load magnetic field data calculated by the magnetic circuit can be verified, so as to adjust the size of the permanent magnet. After processing, the distribution of no-load magnetic field lines and magnetic cloud of the prototype is shown in Fig. 5 and 6.

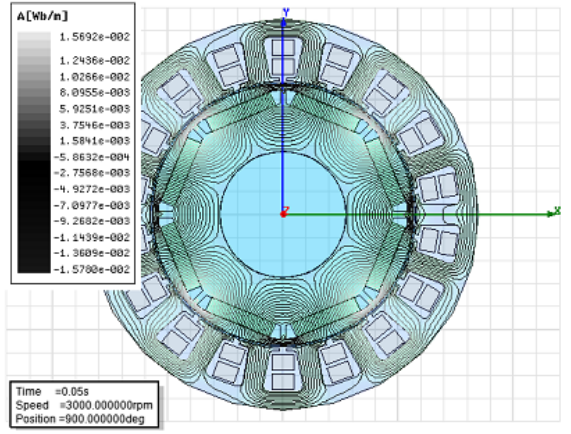

Fig. 5 Distribution of no load magnetic field line of motor

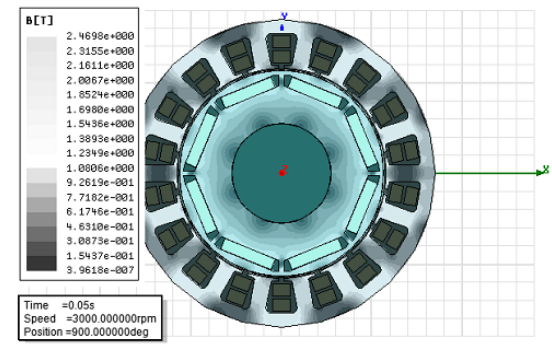

Fig. 6 Magnetic cloud

It can be found in Fig. 5 that there are a small amount of flux lines in the stator slot and the magnetic bridge, which indicates that there is a very small amount of flux leakage in the motor, so the design of the motor is reasonable. In Fig. 6, we can see the magnetic density of each part of the motor intuitively from the distribution of each colour. Due to the existence of the magnetic separation bridge, there is a local saturation phenomenon, and the magnetic flux of the magnetic separation bridge reaches saturation through the magnetic bridge to limit the magnetic flux leakage. There is no obvious saturation phenomenon in stator teeth and stator tooth ends. It shows that the rotor magnetic circuit design of the motor is reasonable, which not only ensures the utilization of motor materials, but also ensures that the motor will not appear magnetic saturation.

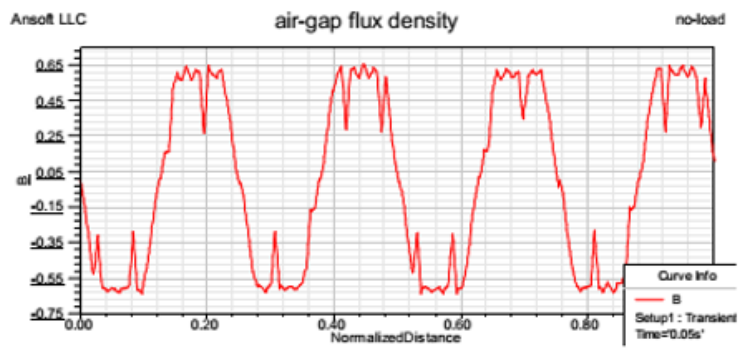

Fig. 7 Magnetic density curve of no-load air gap of motor

The Fig. 7 above shows the magnetic density curve of no-load air gap of the motor. Compared with the standard sine wave, it can be found that the air gap magnetic field of the motor is affected by harmonic and cogging effect. Because the motor adopts the design of less slots, the effect of cogging on the air gap magnetic field can be seen obviously. The Fourier decomposition of the gap magnetic density curve is shown in the following Fig. 8. 


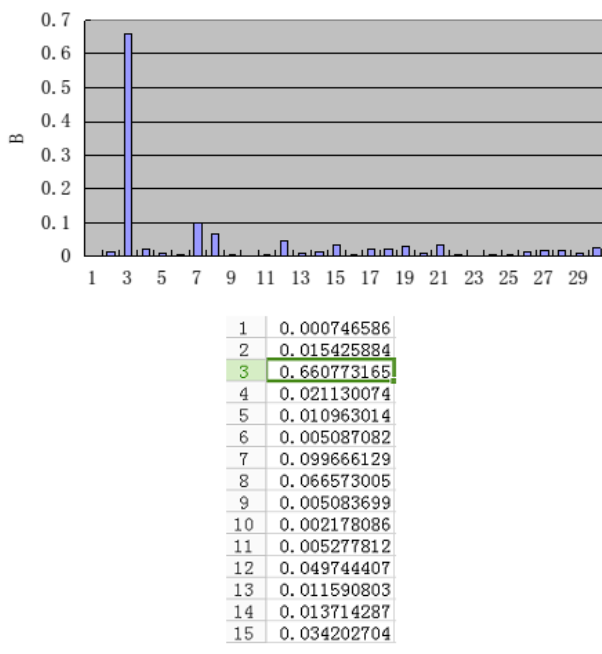

Fig. 8 Fourier decomposition of motor no load air gap flux density curve

From the results of Fourier decomposition, the harmonic suppression of the motor is better, and the fundamental amplitude is $0.6608 \mathrm{t}$. In the simulation postprocessing of motor no-load magnetic field, we also get the no-load counter EMF waveform of the motor, as shown in Fig. 9 below. Compared with the standard sine wave, the voltage waveform distortion in the figure is very small. It shows that the double-layer fractional winding structure greatly weakens the harmonic electromotive force.

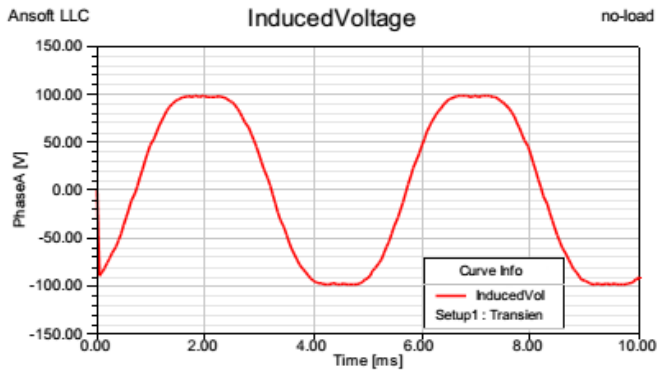

Fig. 9 No load counter EMF curve of motor

Next, we put the rated load on the motor. The load torque curve of the motor (Fig. 10) shows that the torque ripple in the torque stable section fluctuates between 54 $56 \mathrm{~nm}$.

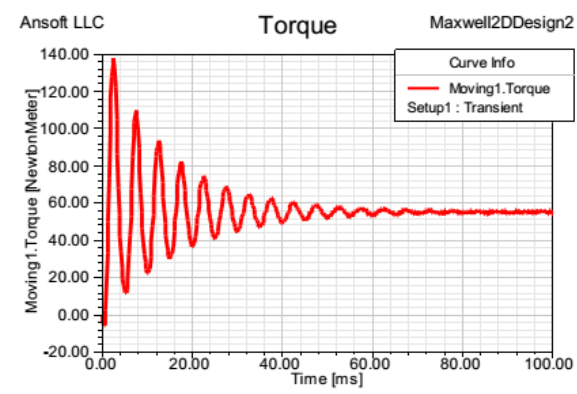

Fig. 10 Motor load torque curve

To verify whether the output power meet the design requirements, we use the formula as below:

$$
p_{\text {out }}=T_{a v} \Omega=T_{a v} \frac{2 \pi n}{60}
$$

Where Tav is the average torque of the motor (nm); $\Omega$ is the motor rotation angle speed $(\mathrm{rad} / \mathrm{s}) ; \mathrm{n}$ is the motor speed (RPM). The average value of torque is $55 \mathrm{~nm}$. The rated speed of the motor is $3000 \mathrm{rpm}$. The output power of the motor is $17.3 \mathrm{kw}$ by substituting the above formula, which meet the design requirements.

\section{Conclusion}

In this chapter, through the finite element analysis, it is verified that the magnetic circuit design and working performance of the motor can meet the design requirements.

The magnetic field of the no-load PM motor is obtained by simulation. After data post-processing, the air gap magnetic field and the electromotive force waveform are analyzed, which can meet the design requirements.

Through the magnetic field simulation of the motor under load, the magnetic field data of the motor after armature reaction is obtained. After data processing, the output torque ripple of the motor is analysed, and the output power of the motor is calculated, which can meet the design requirements.

\section{References}

1. SR Jiang, Urban Transport of China, 10(2), 66-69, (2003).

2. QY Zhang, XS Zhou, JL Tan, Electric Machines \& Control Application, 10(8), 33-38, (2003)

3. B Deng, JM Pan, Computer Simulation, 9(4), 25-30, (2002).

4. CC Chan, KT chou, IEEE Translation on Ind. Electron, 44(1), 29-30, (1997)

5. W A Lynch, IEEE Translation on Energy conversion, 12,407-412, (1997).

6. G Liu, HY Wang, Shanghai Auto, 15(12), 22-27, (2005). 